\title{
Novel Therapies Potentially Available for Pediatric B-Cell Non-Hodgkin Lymphoma
}

\author{
Paul D. Harker-Murray, MD, $\mathrm{PhD}^{1}$; Lauren Pommert, MD, MS'1; and Matthew J. Barth, $\mathrm{MD}^{2}$
}

\section{ABSTRACT}

Burkitt lymphoma, diffuse large B-cell lymphoma (DLBCL), and primary mediastinal B-cell lymphoma are the most common aggressive pediatric mature B-cell non-Hodgkin lymphomas (B-NHLs). Despite excellent survival with current chemotherapy regimens, therapy for Burkitt lymphoma and DLBCL has a high incidence of short- and longterm toxicities. Patients who experience relapse generally have a very poor prognosis. Therefore, novel approaches using targeted therapies to reduce toxicities and improve outcomes in the relapse setting are needed. The addition of rituximab, a monoclonal antibody against CD20, to upfront therapy has improved survival outcomes for highrisk patients and may allow decreased total chemotherapy in those with low-risk disease. Antibody-drug conjugates have been combined with chemotherapy in relapsed/refractory (R/R) NHL, and multiple antibody-drug conjugates are in development. Additionally, bispecific T-cell-engaging antibody constructs and autologous CAR T-cells have been successful in the treatment of $R / R$ acute leukemias and are now being applied to R/R B-NHL with some successes. PD-L1 and PD-L2 on tumor cells can be targeted with checkpoint inhibitors, which restore T-cell-mediated immunity and antitumor responses and can be added to conventional chemotherapy and immune-directed therapies to augment responses. Lastly, trials of small molecule inhibitors targeting cell signaling pathways in NHL subtypes are underway. This article reviews many of the targeted therapies under development that could be considered for future trials in $\mathrm{R} / \mathrm{R}$ pediatric mature $\mathrm{B}-\mathrm{NHL}$.

J Natl Compr Canc Netw 2020;18(8):1125-1134 doi: $10.6004 /$ jnccn.2020.7608

1Pediatric Oncology, Midwest Children's Cancer Center, Milwaukee, Wisconsin; and ${ }^{2}$ Roswell Park Cancer Institute, Buffalo, New York.
As a group, lymphomas are the third most frequent malignancy in pediatric patients. Mature B-cell non-Hodgkin lymphomas (B-NHLs) constitute approximately $60 \%$ of all pediatric NHL diagnoses and $7 \%$ of all pediatric cancers. Most pediatric B-NHLs are Burkitt lymphoma, diffuse large B-cell lymphoma (DLBCL), and primary mediastinal B-cell lymphoma (PMBCL). Each is highly aggressive and has its own unique biology. ${ }^{1-5}$ Additionally, there are rare subtypes, including pediatric-type follicular lymphoma, pediatric marginal zone lymphoma, primary central nervous system (CNS) lymphoma, and posttransplant lymphoproliferative disorder.

Sporadic Burkitt lymphoma accounts for up to $80 \%$ of pediatric B-NHL in developed nations. ${ }^{3,5}$ Burkitt lymphoma is a germinal center mature B-NHL with a phenotype that includes expression of CD10, CD19, CD20, $\mathrm{CD} 22$, surface IgM in $>90 \%$ of cases, and proliferation marker Ki67 in $>90 \%$ of cells. Nearly all constitutively express the MYC oncogene due to translocations with the immunoglobulin heavy chain $[\mathrm{t}(8 ; 14)(\mathrm{q} 24 ; \mathrm{q} 32)]$ in $80 \%$ of cases, or the kappa light chain $[\mathrm{t}(2 ; 8)(\mathrm{p} 11 ; \mathrm{q} 24)]$ or lambda light chain $[\mathrm{t}(8 ; 22)(\mathrm{q} 24 ; \mathrm{q} 11)]$ in $15 \%$ and $5 \%$, respectively. Additionally, MYC-positive cases frequently have a mutation in one of the genes in the ID3-TCF-CCND3 pathway, which may account for a second hit in Burkitt lymphoma pathogenesis. ${ }^{6}$ MYC-negative Burkitt-like lymphoma with 11q aberrations is a provisional entity in the 2016 WHO lymphoid classification.

DLBCL accounts for $10 \%$ to $20 \%$ of pediatric B-NHL. ${ }^{2,3,5}$ Phenotypically, DLBCLs often express light chain-restricted surface IgG as well as B-cell markers CD19, CD20, CD22, CD79a, PAX-5, and occasionally CD30, which is a nonspecific marker of activation. Expression of the proliferation marker Ki-67 is lower than in Burkitt lymphoma, usually $<90 \%$. In addition, one-third express the MYC transcription factor, which plays an important role in tumor pathogenesis and development, ${ }^{7}$ and two-thirds express the antiapoptosis protein BCL2. Coexpression of these 2 proteins occurs in approximately $10 \%$ of germinal center DLBCL and is associated with worse prognosis. ${ }^{8}$ Gene expression profiling has demonstrated that most pediatric DLBCLs are the germinal center B-cell subtype (GCB) rather than the activated B-cell subtype (ABC) more commonly seen in adults. This difference in 
underlying biology somewhat limits the ability to extrapolate the results of mature B-NHL trials in adults to pediatric patients.

PMBCL accounts for only $3 \%$ of pediatric NHL diagnoses, and unlike Burkitt lymphoma and DLBCL, it is a thymic B-cell lymphoma. ${ }^{2-4,9}$ Phenotypically, PMBCL expresses B-cell markers, including CD19, CD20, CD22, and CD79a, but not surface immunoglobulin. It has a unique gene expression profile and genomic changes that overlap with classic Hodgkin lymphoma, including constitutive activation of the NF- $\mathrm{BB}$ and JAK-STAT pathways, immune evasion via downregulation of MHC I and II, and alterations in the 9p24 locus resulting in PD-L1 and PD-L2 expression.

\section{Current Therapies Combine Monoclonal Antibodies With Chemotherapy}

The International Pediatric NHL Staging System ${ }^{10}$ is based on the Murphy (St. Jude) criteria ${ }^{11}$ and allows for allocation of pediatric patients with Burkitt lymphoma and DLBCL into risk groups based on disease stage with or without resection in the French-American-British (FAB) / Lymphome Malins B (LMB) protocols and with the additional incorporation of lactate dehydrogenase level in the Berlin-Frankfurt-Münster (BFM) protocols. In the prerituximab era, risk-stratified, response-based, multiagent chemotherapy included higher doses and additional cycles of chemotherapy for patients with higher-stage disease and resulted in cure rates ranging from $79 \%$ to $98 \%$ (Table 1).

The addition of immunotherapy has increased survival in patients with higher-stage disease. Rituximab is a chimeric murine/human monoclonal antibody with a high affinity against CD20 on B cells that functions through complement-mediated lysis and antibodydependent cell-mediated cytotoxicity (ADCC). The addition of rituximab to standard chemotherapy improved 3-year event-free survival (EFS) rates from $59 \%$ (95\% CI, $54 \%-64 \%)$ to $79 \%$ (95\% CI, $75 \%-83 \%)(P<.0001)$ in adults with DLBCL. ${ }^{12}$ In a phase II window study of pediatric patients with newly diagnosed B-NHL, rituximab monotherapy demonstrated a response rate of $41.4 \%(95 \% \mathrm{CI}, 31 \%-52 \%)$ measured 5 days after administration. ${ }^{13}$ Rituximab was shown to be safe when combined with standard chemotherapy in a Children's Oncology Group (COG) pilot study, and resulted in 3-year EFS rates of $95 \%$ (95\% CI, 80\%-99\%) for intermediate-risk FAB group B (stage III/IV) patients ${ }^{14}$ and $90 \%(95 \%$ CI, 76\%-96\%) for high-risk group C patients. In this study, outcomes were comparable for patients with and without CNS involvement. ${ }^{15,16}$ Most recently, the European Intergroup/COG Inter-B-NHL 2010 trial, which randomized high-risk patients with Burkitt lymphoma and DLBCL to chemotherapy with or without rituximab, was terminated early due to an increased 1-year EFS rate of $94.2 \%(95 \% \mathrm{CI}, 88.5 \%-97.2 \%)$ in the rituximab arm compared with $81.5 \%$ (95\% CI, $73.0 \%-87.8 \%$ ) in the control arm. ${ }^{17}$ Based on the outcomes after the addition of rituximab in higher-stage NHL, it could be inferred that that rituximab should be added to standard chemotherapy backbones for patients with lower-stage disease as well, although this has not been investigated in a clinical trial.

Despite excellent EFS with current chemotherapy regimens, patients with Burkitt lymphoma and DLBCL have a high incidence of grade 3-4 oral mucositis,

\section{Table 1. Outcomes for Current Chemotherapy Regimens in Pediatric Burkitt Lymphoma and DLBCL}

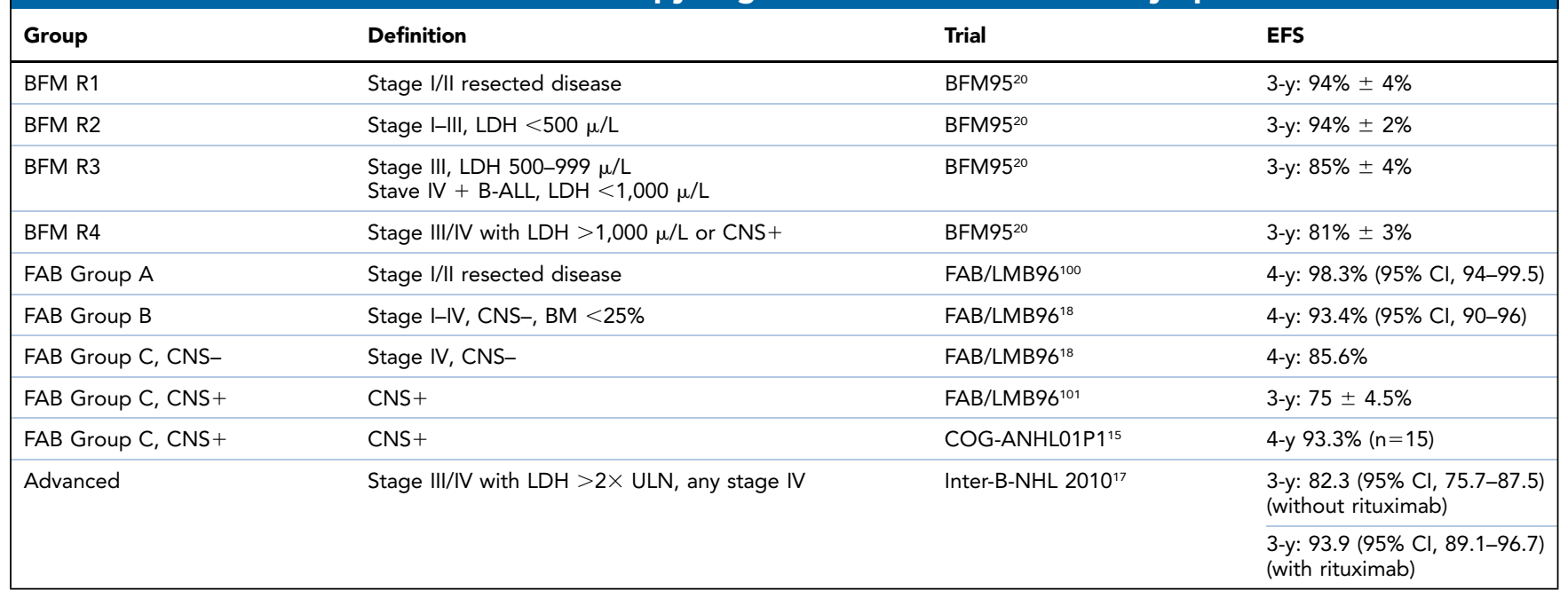

Abbreviations: B-ALL, B-cell acute lymphoblastic leukemia; B-NHL, B-cell non-Hodgkin lymphoma; BFM, Berlin-Frankfurt-Münster; BM, bone marrow; CNS, central nervous system; EFS, event-free survival; FAB, French-American-British; LDH, lactate dehydrogenase; ULN, upper limit of normal. 
hematologic toxicities, and infections, which often result in prolonged hospitalizations. In addition, other longterm effects of current chemotherapy regimens include risk of infertility, secondary malignancies, and late cardiac toxicity due to high cumulative doses of alkylators, topoisomerase II inhibitors, and anthracyclines. Novel approaches to reduce both acute and long-term toxicities are needed. The FAB/LMB96 trial demonstrated that for patients with intermediate-risk B-NHL who had an early response to therapy and achieved a complete remission (CR) after their first consolidation course, the cumulative cyclophosphamide dose could be safely reduced and the maintenance 1 phase could be omitted. ${ }^{18}$ The FAB/LMB96 trial also replaced CNS irradiation with high-dose methotrexate and had similar outcomes to the prior LMB89 trial. ${ }^{18,19}$ The NHL-BFM95 trial demonstrated that reducing high-dose methotrexate dose and infusion rate reduced the incidence of grade III/IV mucositis. ${ }^{20}$ Highdose methotrexate is not used in adult DLBCL and could be a future target for replacement by a less toxic agent. The most recent NHL-BFM and Scandinavian NOPHO pediatric B-NHL trial (B-NHL 2013) is testing whether rituximab can be substituted for anthracyclines in patients with limited-stage disease (R1, R2 stage 1 and II) without compromising survival, as well as analyzing the survival impact of adding rituximab to chemotherapy for patients with higher-stage disease (ClinicalTrials.gov identifier: NCT03206671). A recently closed clinical trial from the New York Medical College is investigating whether the addition of rituximab and liposomal cytarabine to the FAB Group B backbone can safely reduce the cumulative anthracycline dose and number of intrathecal injections for a subset of patients (NCT01859819). ${ }^{21}$

Rituximab has been added to chemotherapy for PMBCL as a substitute for mediastinal radiation, ${ }^{22,23}$ thus reducing the risk of cardiotoxicity and breast cancer. In a phase II trial of 51 adults with PMBCL, the addition of rituximab to dose-adjusted etoposide/doxorubicin/ cyclophosphamide + vincristine/prednisone (DA-EPOCH) increased EFS from $70 \%{ }^{24}$ to $93 \% .^{23}$ In pediatric PMBCL, chemotherapy regimens successful in pediatric Burkitt lymphoma and DLBCL have resulted in EFS of only approximately $66 \% .{ }^{25}$ Therefore, the adult strategy of DAEPOCH-R was applied to pediatric patients with PMBCL in a prospective trial of 47 patients but resulted in a 2 -year EFS rate of only $72 \%$ (95\% CI, $57 \%-84 \%) .{ }^{26}$ In a retrospective review of 156 patients, including 38 pediatric patients, treated with DA-EPOCH-R at 24 academic centers, 3-year EFS was $86 \%$ (95\% CI, $80.3 \%-91.5 \%) .{ }^{27}$ Although pediatric EFS rates are less than those seen in adults, the DA-EPOCH-R regimen (without radiation) has fewer acute toxicities, including less stomatitis, enteritis, febrile neutropenia, and infections, compared with other pediatric B-NHL chemotherapy backbones, ${ }^{17,23}$ and has been adopted as the standard therapy for this group of patients.

\section{Relapsed B-NHL}

Relapsed pediatric B-NHL has a very poor prognosis with survival rates ranging from $27 \%$ to $36 \% .^{28-30}$ Common chemotherapy salvage approaches include rituximab + ifosfamide/carboplatin/etoposide (R-ICE) or rituximab + high-dose cytarabine/etoposide (R-CYVE). These salvage regimens have CR rates of $35 \%$ to $47 \%$ and are usually followed by either autologous or allogeneic hematopoietic stem cell transplantation (HSCT). In a prospective COG study of relapsed B-NHL, only 7 of 20 patients were alive and disease-free, and 5 of the 6 patients who underwent HSCT (4 autologous, 2 allogeneic) were survivors. ${ }^{29}$ In a retrospective study in the United Kingdom and Ireland, only 9 of 27 patients survived, all of whom received HSCT (8 autologous, 1 allogeneic) in CR. ${ }^{28}$ Similarly, in a retrospective study of 33 patients in France, 5-year survival for patients in CR at the time of transplant was 75\% (95\% CI, 46.8\%-91.1\%) compared with 33.3\% (95\% CI, 9.7\%-70\%) for patients who underwent HSCT following only a partial response (PR). ${ }^{30}$ Additionally, this study found no difference in survival based on type of transplant. Therefore, although HSCT in CR remains standard, relapse post HSCT is common, and it is unclear whether autologous or allogeneic HSCT provides superior disease control. ${ }^{31,32}$ Two smaller trials have shown promising results using myeloablative autologous HSCT followed by reducedintensity conditioning allogeneic HSCT. ${ }^{30,33,34}$ There are no case series of relapsed pediatric PMBCL; however, in adult patients with relapsed PMBCL, even with allogeneic HSCT progression-free survival is only $34 \% .^{35}$ Therefore, novel strategies are needed for pediatric patients with relapsed mature B-NHL, especially for those who experience relapse posttransplant. Although there are many agents being developed for B-NHL in adults (Table 2), relatively few are being tested in pediatric patients. Selection of novel therapies for pediatric clinical trials will likely be derived from clinical trials that are successful in adults (Table 3).

\section{Monoclonal Antibodies}

Multiagent chemotherapy with rituximab has become the standard of care for most intermediate- and high-risk pediatric mature B-NHL. A number of next-generation monoclonal antibodies have also been investigated but have had limited success in improving survival outcomes. Obinutuzumab, a type II monoclonal antibody with greater in vitro ADCC and direct cell death than rituximab, ${ }^{36}$ did not improve outcomes in adults newly diagnosed with DLBCL randomized to receive either rituximab or obinutuzumab in conjunction with cyclophosphamide/ doxorubicin/vincristine/prednisone (CHOP) ${ }^{37}$ A pediatric 


\begin{tabular}{|c|c|c|}
\hline Class & Medication & Target \\
\hline \multirow{6}{*}{$\begin{array}{l}\text { Antibody drug } \\
\text { conjugate }\end{array}$} & Inotuzumab ozogamicin & $\mathrm{CD} 22$ \\
\hline & Pinatuzumab vedotin & CD22 \\
\hline & Brentuximab vedotin & CD30 \\
\hline & Polatuzumab vedotin & CD79b \\
\hline & Denintuzumab mafodotin & CD19 \\
\hline & Debio 15621 & CD37 \\
\hline \multirow[t]{4}{*}{ Monoclonal antibody } & Rituximab & CD20 \\
\hline & Obinutuzumab & CD20 \\
\hline & Ofatumumab & CD20 \\
\hline & Veltuzumab & CD20 \\
\hline \multirow[t]{4}{*}{ T-cell engagers } & Blinatumomab & CD19 \\
\hline & Mosunetuzumab & CD20 \\
\hline & REGN1979 & CD20 \\
\hline & RG6026 & CD20 \\
\hline \multirow[t]{5}{*}{ CAR T-cells } & CTL019 (tisagenlecleucel) & CD19 \\
\hline & $\begin{array}{l}\text { KTE-C19 (axicabtagene } \\
\text { ciloleucel) }\end{array}$ & CD19 \\
\hline & Lisocabtagene maraleucel & CD19 \\
\hline & MB-106 & CD20 \\
\hline & PBCAR0191 & CD19 \\
\hline \multirow[t]{4}{*}{ Checkpoint inhibitors } & Nivolumab & PD-1 \\
\hline & Ipilimumab & CTLA-4 \\
\hline & Pembrolizumab & PD-1 \\
\hline & Pidilizumab & PD-1/DLL1 \\
\hline \multirow{15}{*}{$\begin{array}{l}\text { Cell signaling } \\
\text { inhibitors }\end{array}$} & Ibrutinib & BTK \\
\hline & Acalabrutinib & BTK \\
\hline & Buparlisib & PI3K \\
\hline & Copanlisib & PI3K \\
\hline & Idelalisib & PI3K \\
\hline & IPI-145 & PI3K \\
\hline & Temsirolimus & mTOR \\
\hline & Everolimus & mTOR \\
\hline & Bortezomib & $26 \mathrm{~S}$ proteosome \\
\hline & Venetoclax & $\mathrm{BCL}-2$ \\
\hline & Navitoclax & $\mathrm{BCL}-2$ \\
\hline & Lenalidomide & IRF4 \\
\hline & EPZ-6438 & $\mathrm{EZH} 2$ \\
\hline & GSK2816126 & $\mathrm{EZH} 2$ \\
\hline & CPI-1205 & EZH2 \\
\hline
\end{tabular}

phase II trial combining obinutuzumab + ifosfamide/ carboplatin/etoposide (ICE) is ongoing (ClinicalTrials.gov identifier: NCT02393157). In a clinical trial that closed early due to slow accrual, the second-generation
anti-CD20 human monoclonal antibody ofatumumab resulted in 2 patients with PR and 2 patients with stable disease among 11 enrolled patients. ${ }^{38}$ Similar response rates were seen when ofatumumab was combined with lenalidamide. ${ }^{39}$ No difference was seen in EFS for patients with R/R DLBCL randomized to either ofatumumab or rituximab in addition to cisplatin/cytarabine/ dexamethasone (DHAP). ${ }^{40}$ The slow onset of effect, lack of improved outcomes using monoclonal antibodies against the same antigen, and fact that most patients with R/R B-NHL have previously received CD20-directed therapy at initial diagnosis have limited the enthusiasm for future clinical trials using next-generation monoclonal antibodies in pediatric B-NHL. ${ }^{41}$

\section{Antibody-Drug Conjugates}

The conjugation of monoclonal antibodies to agents that are cytotoxic at nanomolar concentrations allows increased tumor-specific drug delivery, higher intracellular drug concentrations, and increased potency compared with traditional chemotherapy. ${ }^{42,43}$ Approximately $30 \%$ of DLBCL $^{44}$ and $80 \%$ of PMBCL ${ }^{45}$ express CD30, a member of the tumor necrosis factor receptor superfamily and an inducible marker of activation. ${ }^{44}$ Brentuximab vedotin $(\mathrm{Bv})$ is a monoclonal anti-CD30 antibody conjugated with monomethyl auristatin E (MMAE) with established pediatric safety data. ${ }^{4-48}$ In an adult phase II study of 44 patients with R/R CD30+ DLBCL, there was an overall response rate (ORR) of $44 \%$ (95\% CI, $29.5 \%-50.8 \%$ ) with 8 CRs $(15 \%)$ and 13 PRs (27\%). ${ }^{49}$ This study also showed a similar ORR of $31 \%(95 \%$ CI, $18.7 \%-45.1 \%)$ in patients with undetectable CD30 expression by conventional immunohistochemistry, with 6 (12\%) CR and 10 (19\%) PR, ${ }^{50}$ suggesting a cytotoxic mechanism that is independent of CD30 expression. A phase II study of $\mathrm{Bv}$ in adults with $\mathrm{R} / \mathrm{R}$ PMBCL showed no CRs and a PR in only 2 of 15 patients. ${ }^{51}$

Inotuzumab ozogamicin (IO) is a humanized antiCD22 IgG4 conjugated with the cytotoxic antibiotic calicheamicin and demonstrated only modest responses (2 CRs, 2 PRs) in the phase I trial of 26 adults patients with $\mathrm{R} / \mathrm{R}$ DLBCL. ${ }^{52}$ However, CR rates were $30 \%$ to $50 \%$ in subsequent studies that combined IO with rituximab. ${ }^{53,54}$ The higher-than-expected incidence of cytopenias and sinusoidal obstruction syndrome (SOS) suggests off-target activity of the drug. IO was very well tolerated in the prospective phase II trial of 49 pediatric patients with $\mathrm{R} / \mathrm{R}$ CD22+ acute lymphoblastic leukemia (ALL); however, in this study, the incidence of SOS in patients who underwent allogeneic HSCT was 40\% (4 of 10 patients) (ClinicalTrials.gov identifier: NCT02981628). Similarly, in a retrospective review of 51 pediatric patients with R/R ALL who received IO, no cases of SOS occurred while patients were receiving the drug; however, 11 of 
Table 3. Recent Adult Clinical Trials Using Novel Therapies for DLBCL

\begin{tabular}{|c|c|c|c|c|c|c|c|}
\hline Category & Medications & Target & Phase & Diagnosis & No. Treated & CR n (\%) & PR n (\%) \\
\hline \multirow{8}{*}{ ADC combinations } & Inotuzumab + rituximab ${ }^{53}$ & $\mathrm{CD} 22+\mathrm{CD} 20$ & $1 / I I$ & $\mathrm{R} / \mathrm{R} \mathrm{DLBCL}$ & 42 & $21(50 \%)$ & $10(23.8 \%)$ \\
\hline & Inotuzumab + rituximab ${ }^{102}$ & $\mathrm{CD} 22+\mathrm{CD} 20$ & III & R/R DLBCL & 166 & $26(16 \%)$ & $42(25 \%)$ \\
\hline & Polatuzumab ${ }^{56}$ & CD79b & 1 & R/R DLBCL & 25 & $4(16 \%)$ & $10(40 \%)$ \\
\hline & Polatuzumab + rituximab 60 & $\mathrm{CD} 79 \mathrm{~b}+\mathrm{CD} 20$ & ॥ & R/R DLBCL & 39 & $8(20.5 \%)$ & $13(33.3 \%)$ \\
\hline & Obinutuzumab + $\mathrm{CHOP}^{37}$ & CD20 & III & DLBCL & 706 & $248(35.1 \%)$ & $329(46.6 \%)$ \\
\hline & Polatuzumab + obinutuzumab ${ }^{57}$ & $C D 79 b+C D 20$ & $\mathrm{Ib} / \mathrm{ll}$ & $\mathrm{R} / \mathrm{R} \mathrm{DLBCL}$ & 21 & $6(28.6 \%)$ & $5(23.8 \%)$ \\
\hline & Pinatuzumab + rituximab 60 & $C D 79 b+C D 20$ & II & $\mathrm{R} / \mathrm{R} \mathrm{DLBCL}$ & 42 & $11(26.2 \%)$ & $14(33.3 \%)$ \\
\hline & Polatuzumab + bendamustine + rituximab $^{58}$ & $\mathrm{CD} 79 \mathrm{~b}+\mathrm{CD} 20$ & II & $\mathrm{R} / \mathrm{R} \mathrm{DLBCL}$ & 40 & $23(57.5 \%)$ & $5(12.5 \%)$ \\
\hline \multirow[t]{3}{*}{ Immunotherapy } & Blinatumomab 66 & CD19 & II & R/R DLBCL & 21 & $4(19 \%)$ & $5(23.8 \%)$ \\
\hline & Mosunetuzumab ${ }^{67}$ & CD20 & $\mathrm{l} / \mathrm{lb}$ & $\mathrm{R} / \mathrm{R} \mathrm{DLBCL}$ & 73 & $13(17.8 \%)$ & $11(15 \%)$ \\
\hline & REGN197969 & CD20 & 1 & $\mathrm{R} / \mathrm{R} \mathrm{DLBCL}$ & 19 & $8(42.1 \%)$ & $3(15.8 \%)$ \\
\hline \multirow[t]{2}{*}{ CAR T-cells } & Axicabtagene ciloleuce $\left.\right|^{73}$ & CD19 & ॥ & $\mathrm{R} / \mathrm{R} \mathrm{DLBCL}$ & 77 & $38(49 \%)$ & $25(32 \%)$ \\
\hline & CTL019 (tisagenlecleucel) $^{72}$ & CD19 & Ila & R/R DLBCL & 93 & 48 (52\%) & 37 (40\%) \\
\hline
\end{tabular}

Abbreviations: $A D C$, antibody-drug conjugate; $C H O P$, cyclophosphamide/doxorubicin/vincristine/prednisone; $C R$, complete response; DLBCL, diffuse large B-cell lymphoma; G-CHP, obinutuzumab/cyclophosphamide/doxorubicin/prednisone; NR, not reported; PR, partial response; R-CHP, rituximab/cyclophosphamide/ doxorubicin/prednisone; R/R, relapsed/refractory.

21 patients $(52.4 \%)$ who subsequently underwent allogeneic HSCT developed SOS during transplant. ${ }^{55}$

CD79B, a component of the B-cell antigen receptor, is expressed on most B-cell-derived malignancies. ${ }^{56}$ Polatuzumab vedotin is a CD79B-directed monoclonal antibody conjugated with MMAE and induces cell death by microtubule inhibition, antibody-mediated opsonization, and ADCC. ${ }^{43}$ In the adult phase I trial of 25 patients with R/R DLBCL treated at the recommended phase II dosing, there were 4 CRs and 10 PRs. ${ }^{56}$ Polatuzumab vedotin has subsequently been combined with rituximab, ${ }^{56}$ obinutuzumab, ${ }^{57}$ and bendamustine and rituximab or obinutuzumab ${ }^{58}$ for patients with R/R DLBCL. In the latter study, ${ }^{58}$ with a mean follow-up of 30 months, 9 of 19 responders remained event-free. In previously untreated adult patients, polatuzumab combined with cyclophosphamide/doxorubicin/prednisone and either rituximab or obinutuzumab had an $89 \%$ ORR, a $77 \%$ CR rate, and a 24-month progression-free survival of $83 \%$ (95\% CI, 73-93). ${ }^{59}$

Pinatuzumab vedotin is an anti-CD22 ADC conjugated to MMAE. In the ROMULUS trial, adults with R/R B-NHL were randomized to rituximab and either pinatuzumab vedotin or polatuzumab vedotin with comparable outcomes ${ }^{60}$; however, polatuzumab vedotin was chosen for further development due to longer durations of response and fewer toxicities. ${ }^{60}$ Because immunotherapy has improved outcomes in both adult and pediatric B-NHL trials, ADCs may show increased efficacy in patients with prior exposure to naked monoclonal antibodies.

\section{T-Cell Engagers}

Bispecific T-cell-engaging antibody constructs simultaneously bind to tumor-specific surface antigens and CD3, a component of the T-cell receptor complex on cytotoxic $\mathrm{T}$ lymphocytes (CTLs) which results in CTL activation, formation of cytolytic synapses, and tumor cell lysis. ${ }^{61}$ Blinatumomab is a CD19/CD3 bispecific antibody that is active in adults ${ }^{62}$ and children ${ }^{63,64}$ with R/R ALL and was superior to chemotherapy in the relapse setting in adults. ${ }^{65}$ Blinatumomab was also active in a small trial in adults with heavily pretreated R/R DLBCL, in which 4 of 18 evaluable patients had a CR and 5 a PR. ${ }^{66}$ Mosunetuzumab is a full-length, fully humanized CD20/CD3 bispecific IgG1 antibody that induced CR in 13 of 73 adults with R/R DLBCL, ${ }^{67}$ including 4 of 16 patients who had received prior CAR T-cell therapy. ${ }^{68}$ REGN1979 is a CD20/CD3 bispecific IgG4 modified to reduce Fc binding. In a phase I trial in heavily pretreated adults with $R / R$ 
DLBCL, 5 of 7 patients without prior CAR-T exposure achieved CR compared with CR in 3 of 12 patients with prior CAR T-cell therapy. ${ }^{69}$ A large phase II study of REGN1979 in R/R DLBCL is ongoing (ClinicalTrials. gov identifier: NCT03888105). Safety and efficacy data for blinatumomab are known for pediatric patients with R/R ALL ${ }^{63,64}$; however, neither mosunetuzumab or REGN1979 have been used in children, nor are there published bispecific antibody data in pediatric NHL.

\section{CAR T-Cell Therapy}

CAR T-cells are autologous T cells engineered to express single-chain variable fragment antigen recognition moiety, a transmembrane linker, and a CD3 $\zeta$ intracellular activation domain that triggers cytotoxic effect. ${ }^{70} \mathrm{CAR}$ T-cells bind specific tumor surface antigens in a human leukocyte antigen-independent manner that combines the specificity of monoclonal antibodies with the cytotoxic effector function of CTLs and has the potential for in vivo expansion and persistence. Tisagenlecleucel is a second-generation CD19 CAR-T with an intracellular 4-1BB costimulatory domain designed to provide improved cytokine production, proliferation, and CAR-T persistence, and is highly active in children with $R / R$ ALL. ${ }^{71}$ In a phase II trial of tisagenlecleucel for $R / R$ DLBCL, $40 \%$ of patients achieved CR and the 2-year relapse-free survival was estimated to be $65 \%$ for all patients and $79 \%$ for those who achieved CR. ${ }^{72}$ A phase II trial of tisagenlecleucel for pediatric R/R NHL is ongoing (ClinicalTrials.gov identifier: NCT03610724). Axicabtagene ciloleucel is a CD19 CAR-T with a CD28 costimulatory domain and has been shown to have activity in adults with R/R DLBCL. In a phase II trial of 101 adults who received axicabtagene ciloleucel, those with $R / R$ DLBCL had a $49 \%$ CR rate. ${ }^{73}$ At a median follow-up of 15.4 months, CR was sustained in $40 \%$ of all patients (including those with PMBCL and transformed follicular lymphoma). Clinical trials using lisocabtagene maraleucel, a CD19 CAR-T with a 4-1BB costimulatory domain ${ }^{74}$; MB106, a third-generation CD20 CAR-T with intracellular 4-1BB and CD28 domains ${ }^{75}$; and PBCAR0191, an allogeneic CD19 CAR-T cell designed for "off the shelf" use ${ }^{76}$ are in early-phase trials in adults. Severe cytokine-release syndrome and neurotoxicity have the potential to limit the therapeutic application of CAR-T therapies, and novel constructs are being designed to reduce these toxicities while maintaining cytolytic activity. ${ }^{77}$ Early pediatric trials in relapsed CD19+ B-NHL are also ongoing. ${ }^{78,79}$

\section{Targeted Therapies}

A number of cell-signaling inhibitors are being tested in early-phase clinical trials. ABC DLBCL acquires mutations that result in chronic BCR signaling. Ibrutinib, a selective covalent inhibitor of Bruton tyrosine kinase (BTK), inhibits BCR signaling and is active in adults with ABC DLBCL, but much less so in GCB DLBCL. ${ }^{80,81}$ Although most pediatric DLBCL is the GCB type, ibrutinib is currently being combined with chemotherapy in pediatric patients with $R / R$ mature NHL (ClinicalTrials.gov identifier: NCT02703272). A number of ongoing clinical trials in adults are combining chemotherapy with either ibrutinib or the second-generation BTK inhibitor acalabrutinib, which has greater selectivity and potency. ${ }^{82}$ Another potential signaling target is the phosphoinositide 3-kinase (PI3-K pathway), which appears to be active in preclinical studies of Burkitt lymphoma ${ }^{83}$; however, data showing efficacy of PI3-K inhibitors in aggressive B-NHL are lacking. ${ }^{84}$ The antiapoptotic protein BCL2 is overexpressed in a subset of patients with DLBCL and is associated with inferior prognosis. Venetoclax is a BCL2 inhibitor that is active in adults with R/R DLBCL $L^{85}$ and may function as a chemosensitizing agent. ${ }^{86,87}$ Lenalidomide is an oral immune-modulating medication that targets the E3 ubiquitin ligase cereblon and preclinically exhibited synergy with rituximab. ${ }^{88}$ It has also been found to be effective in adult-type ABC DLBCL when combined with rituximab and R-CHOP; however, these subtypes are rarely found in pediatric patients. ${ }^{89-91}$ Bortezomib is a proteasome inhibitor that targets the NF-kB pathway and can induce apoptosis in tumor cells that continuously express NF-kB. A meta-analysis showed that the bortezomib-containing regimens did not prolong survival in patients with ABC DLBCL. ${ }^{92}$ Although a number of drugs targeting cell signaling pathways exist, enthusiasm is limited by slow onset of effect and the knowledge that each pathway is active in only a subset of pediatric NHL. Therefore, development of a clinical trial of any individual agent would be limited to the relevant NHL subtype.

\section{Checkpoint Inhibitors}

Expression of PD-L1 and PD-L2 on malignant cells and cells in the tumor microenvironment results in exhaustion of PD-1-expressing tumor-infiltrating $\mathrm{T}$ cells and is a mechanism of tumor evasion in the host immune response. ${ }^{93}$ Checkpoint inhibitors are monoclonal antibodies that interrupt PD-1 receptor-ligand interactions and restore T-cell-mediated immunity and antitumor responses. Abnormalities in 9p.24.1 are common in PMBCL and lead to overexpression of PD-L1 and PDL2. ${ }^{4}$ Nivolumab is a fully humanized IgG4 anti-PD-1 antibody. In a phase II trial of adults with R/R PMBCL, the combination of nivolumab with $\mathrm{Bv}$ resulted in a $43 \%$ complete metabolic response rate ${ }^{94}$ Pembrolizumab is a humanized IgG4 monoclonal antibody targeting PD-1. The combined results of a phase Ib trial for R/R NHL and a phase II trial for R/R PMBCL showed an ORR rate of approximately $50 \%$, with $18 \%$ achieving CR. ${ }^{95}$ The COG 
and National Clinical Trials Network are collaborating in a phase III clinical trial randomizing patients with newly diagnosed PMBCL to chemotherapy with or without pembrolizumab.

Although $<20 \%$ of patients with DLBCL have 9p24.1 alterations, PD-L1 expression in adults with DLBCL is associated with nongerminal center immunophenotype and inferior outcomes. ${ }^{96}$ Although the response rate of $8 \%$ in adults with R/R DLBCL treated with nivolumab was disappointing, ${ }^{97}$ the combination of pembrolizumab with R-CHOP in 30 adults with newly diagnosed DLBCL resulted in a $77 \%$ CR rate, which compared favorably with historical controls. ${ }^{98}$ The addition of a checkpoint inhibitor is thought to augment the ADCC effect of rituximab. Given that PD-L1 expression was associated with improved progression-free survival in this cohort and the fact that none of the 19 patients with tumor PD-L1 expression have experienced disease relapse, a biomarkerdriven approach to therapy may be appropriate. Checkpoint inhibitors are currently being used to augment conventional chemotherapy as well as immune-directed therapies for the treatment of hematologic malignancies.

\section{Future Directions and Challenges in Clinical Trial Design}

Advancing treatment of pediatric mature B-NHL is challenging. Although children with newly diagnosed Burkitt lymphoma and DLBCL have an excellent prognosis, current therapies have a high incidence of grade III/IV infection, hematologic toxicities, and mucositis, and novel approaches to reduce both acute and long-term toxicities are needed. Although several recent studies have attempted to reduce cumulative doses of anthracyclines for lowerrisk patients (ClinicalTrials.gov identifier: NCT03206671) ${ }^{21}$ and reduce the total number of chemotherapy cycles for patients with low-risk disease, ${ }^{17}$ deescalation or substitution of current medications with new ones has been met with resistance due to a lack of effective salvage regimens. ${ }^{41}$ As a result, more attention has been focused on improving outcomes for patients with $R / R$ disease.

Development and execution of clinical trials for children with R/R B-NHL is logistically challenging for several reasons. Selection of novel agents for R/R pediatric NHL is based on results from adult studies in ABC DLBCL (Table 3) and may not be applicable to pediatric NHL due to differences in driving mutations, signaling pathways, and mechanisms of immune evasion. Pediatric trials combining histologic subtypes will likely focus on agents that target shared surface antigens rather than intracellular signaling pathways that are specific to each NHL subtype. R/R pediatric NHL is often rapidly progressive, and novel agents should have a rapid onset of effect and be immediately available without requiring significant production time. Finally, it is estimated that there are $<100 \mathrm{R} / \mathrm{R} \mathrm{B}-\mathrm{NHL}$ cases per year in the United States and Europe combined, ${ }^{99}$ and testing even a single new agent in the relapsed setting will require an international collaboration to enroll a sufficient number of patients. However, through international collaboration between adult and pediatric oncology groups and specific focus on the adolescent and young adult population, we can begin to overcome some of the challenges related to testing new agents for the treatment of R/R NHL and expand our use of targeted and immunomodulating medications for this disease.

Submitted March 16, 2020; accepted for publication June 22, 2020.

Disclosures: Dr. Harker-Murray has disclosed that he has received consultant fees from Regeneron Pharmaceuticals. The remaining authors have disclosed that they have no financial interests, arrangements, or affiliations with the manufacturers of any products discussed in this article or their competitors.

Correspondence: Paul D. Harker-Murray, MD, PhD, Pediatric Oncology, Midwest Children's Cancer Center, 8701 Watertown Plank Road, MFRC3018, Milwaukee, WI 53226. Email: pharker@mcw.edu

\section{References}

1. Pizzo PA, Poplack DG. Principles and Practice of Pediatric Oncology. Wolters Kluwer Health 2015.

2. Dunleavy K, Gross TG. Management of aggressive B-cell NHLs in the AYA population: an adult vs pediatric perspective. Blood 2018;132: 369-375.

3. Egan G, Goldman S, Alexander S. Mature B-NHL in children, adolescents and young adults: current therapeutic approach and emerging treatment strategies. Br J Haematol 2019;185:1071-1085.

4. Lees $\mathrm{C}$, Keane $\mathrm{C}$, Gandhi MK, et al. Biology and therapy of primary mediastinal B-cell lymphoma: current status and future directions. $\mathrm{Br} \mathrm{J}$ Haematol 2019;185:25-41.

5. Giulino-Roth L, Goldman S. Recent molecular and therapeutic advances in B-cell non-Hodgkin lymphoma in children. Br J Haematol 2016;173: 531-544.

6. Rohde M, Bonn BR, Zimmermann M, et al. Relevance of ID3-TCF3CCND3 pathway mutations in pediatric aggressive B-cell lymphoma treated according to the non-Hodgkin Lymphoma Berlin-FrankfurtMünster protocols. Haematologica 2017;102:1091-1098.

7. Xia $Y$, Zhang $X$. The spectrum of MYC alterations in diffuse large B-cell lymphoma [published online February 19, 2020]. Acta Haematol, doi: 10.1159/000505892 https://doi.org/10.1159/000505892

8. Sesques $P$, Johnson NA. Approach to the diagnosis and treatment of high-grade B-cell lymphomas with MYC and BCL2 and/or BCL6 rearrangements. Blood 2017;129:280-288.

9. Giulino-Roth L. How I treat primary mediastinal B-cell lymphoma. Blood 2018;132:782-790

10. Rosolen A, Perkins SL, Pinkerton CR, et al. Revised International Pediatric Non-Hodgkin Lymphoma Staging System. J Clin Oncol 2015;33: 2112-2118.

11. Murphy SB. Classification, staging and end results of treatment of childhood non-Hodgkin's lymphomas: dissimilarities from lymphomas in adults. Semin Oncol 1980;7:332-339.

12. Pfreundschuh $M$, Trümper $L$, Osterborg $A$, et al. CHOP-like chemotherapy plus rituximab versus $\mathrm{CHOP}$-like chemotherapy alone in young patients with good-prognosis diffuse large-B-cell lymphoma: a 
randomised controlled trial by the MabThera International Trial (MInT) Group. Lancet Oncol 2006;7:379-391.

13. Meinhardt A, Burkhardt B, Zimmermann $M$, et al. Phase II window study on rituximab in newly diagnosed pediatric mature B-cell non-Hodgkin's lymphoma and Burkitt leukemia. J Clin Oncol 2010;28:3115-3121.

14. Goldman S, Smith L, Anderson JR, et al. Rituximab and FAB/LMB 96 chemotherapy in children with stage III/IV B-cell non-Hodgkin lymphoma: a Children's Oncology Group report. Leukemia 2013;27: 1174-1177.

15. Frazer JK, Li KJ, Galardy PJ, et al. Excellent outcomes in children and adolescents with $\mathrm{CNS}^{+}$Burkitt lymphoma or other mature B-NHL using only intrathecal and systemic chemoimmunotherapy: results from $\mathrm{FAB}$ / LMB96 and COG ANHL01P1. Br J Haematol 2019;185:374-377.

16. Goldman S, Smith L, Galardy P, et al. Rituximab with chemotherapy in children and adolescents with central nervous system and/or bone marrow-positive Burkitt lymphoma/leukaemia: a Children's Oncology Group Report. Br J Haematol 2014;167:394-401.

17. Minard-Colin V, Aupérin A, Pillon M, et al. Rituximab for high-risk, mature B-cell non-Hodgkin's lymphoma in children. N Engl J Med 2020;382: 2207-2219.

18. Patte C, Auperin A, Gerrard M, et al. Results of the randomized international FAB/LMB96 trial for intermediate risk B-cell non-Hodgkin lymphoma in children and adolescents: it is possible to reduce treatment for the early responding patients. Blood 2007;109:2773-2780.

19. Patte C, Auperin A, Michon J, et al. The Société Française d'Oncologie Pédiatrique LMB89 protocol: highly effective multiagent chemotherapy tailored to the tumor burden and initial response in 561 unselected children with B-cell lymphomas and L3 leukemia. Blood 2001;97: 3370-3379.

20. Woessmann W, Seidemann K, Mann G, et al. The impact of the methotrexate administration schedule and dose in the treatment of children and adolescents with B-cell neoplasms: a report of the BFM Group Study NHL-BFM95. Blood 2005;105:948-958.

21. Goldman S, Hochberg J, Harrison L, et al. Reduced burden of oncologic therapy in children, adolescents and young adults with good risk (GR) CD20+ mature B-cell lymphoma [abstract]. Br J Haematol 2018; 182(Suppl):Abstract 11.

22. Messmer M, Tsai HL, Varadhan $\mathrm{R}$, et al. R-CHOP without radiation in frontline management of primary mediastinal B-cell lymphoma. Leuk Lymphoma 2019;60:1261-1265.

23. Dunleavy K, Pittaluga S, Maeda LS, et al. Dose-adjusted EPOCHrituximab therapy in primary mediastinal B-cell lymphoma. N Engl J Med 2013;368:1408-1416.

24. Wilson $\mathrm{WH}$, Grossbard ML, Pittaluga S, et al. Dose-adjusted EPOCH chemotherapy for untreated large B-cell lymphomas: a pharmacodynamic approach with high efficacy. Blood 2002;99:2685-2693.

25. Gerrard M, Waxman IM, Sposto R, et al. Outcome and pathologic classification of children and adolescents with mediastinal large B-cell lymphoma treated with FAB/LMB96 mature B-NHL therapy. Blood 2013; 121:278-285.

26. Burke GAA, Gross TG, Pillon M, et al. Results of Inter-B-NHL Ritux 2010 phase II study of DA-EPOCH-R for children and adolescents with primary mediastinal large B-cell lymphoma (PMLBL) on behalf of European Intergroup for Childhood Non Hodgkin's Lymphoma (EICNHL) and Children's Oncology Group (COG) [abstract]. Blood 2017;130(Suppl): Abstract 4124

27. Giulino-Roth $\mathrm{L}, \mathrm{O}^{\prime}$ Donohue $\mathrm{T}$, Chen $\mathrm{Z}$, et al. Outcomes of adults and children with primary mediastinal B-cell lymphoma treated with doseadjusted EPOCH-R. Br J Haematol 2017;179:739-747.

28. Anoop P, Sankpal S, Stiller C, et al. Outcome of childhood relapsed or refractory mature B-cell non-Hodgkin lymphoma and acute lymphoblastic leukemia. Leuk Lymphoma 2012;53:1882-1888.

29. Griffin TC, Weitzman S, Weinstein H, et al. A study of rituximab and ifosfamide, carboplatin, and etoposide chemotherapy in children with recurrent/refractory B-cell $(C D 20+)$ non-Hodgkin lymphoma and mature B-cell acute lymphoblastic leukemia: a report from the Children's Oncology Group. Pediatr Blood Cancer 2009;52:177-181.

30. Rigaud C, Auperin A, Jourdain A, et al. Outcome of relapse in children and adolescents with B-cell non-Hodgkin lymphoma and mature acute leukemia: a report from the French LMB study. Pediatr Blood Cancer 2019;66:e27873

31. Giulino-Roth L, Ricafort R, Kernan NA, et al. Ten-year follow-up of pediatric patients with non-Hodgkin lymphoma treated with allogeneic or autologous stem cell transplantation. Pediatr Blood Cancer 2013;60: 2018-2024.
32. Gross TG, Hale GA, He W, et al. Hematopoietic stem cell transplantation for refractory or recurrent non-Hodgkin lymphoma in children and adolescents. Biol Blood Marrow Transplant 2010;16:223-230.

33. Gardenswartz A, Mehta B, El-Mallawany N, et al. Safety and efficacy of myeloablative conditioning autologous stem cell transplantation, targeted immunotherapy, and reduced intensity conditioning allogeneic stem cell transplantation in children, adolescents, and young adults with relapsed/refractory mature b-cell non-Hodgkin lymphoma [abstract]. Biol Blood Marrow Transplant 2019;25(Suppl):S412.

34. Satwani $P$, Jin $Z$, Martin PL, et al. Sequential myeloablative autologous stem cell transplantation and reduced intensity allogeneic hematopoietic cell transplantation is safe and feasible in children, adolescents and young adults with poor-risk refractory or recurrent Hodgkin and nonHodgkin lymphoma. Leukemia 2015;29:448-455.

35. Herrera AF, Chen L, Khajavian $\mathrm{S}$, et al. Allogeneic stem cell transplantation provides durable remission in patients with primary mediastinal large B cell lymphoma. Biol Blood Marrow Transplant 2019;25: 2383-2387.

36. Freeman $\mathrm{CL}$, Sehn LH. A tale of two antibodies: obinutuzumab versus rituximab. Br J Haematol 2018;182:29-45.

37. Vitolo $U$, Trněný $M$, Belada $D$, et al. Obinutuzumab or rituximab plus cyclophosphamide, doxorubicin, vincristine, and prednisone in previously untreated diffuse large B-cell lymphoma. J Clin Oncol 2017;35: 3529-3537

38. Galanina N, Jasielec J, Peace D, et al. Ofatumumab monotherapy in relapsed/refractory diffuse large B-cell non-Hodgkin lymphoma. Leuk Lymphoma 2017;58:752-753.

39. Vose JM, Loberiza FR Jr, Bociek G, et al. Phase I/II trial of ofatumumab/ lenalidomide for patients with relapsed/refractory B-cell non-Hodgkin lymphoma: high response rate in indolent lymphoma. Blood 2012;120: 3692-3692.

40. van Imhoff GW, McMillan A, Matasar MJ, et al. Ofatumumab versus rituximab salvage chemoimmunotherapy in relapsed or refractory diffuse large B-cell lymphoma: the ORCHARRD study. J Clin Oncol 2017;35: 544-551.

41. Pearson ADJ, Scobie N, Norga K, et al. ACCELERATE and European Medicine Agency Paediatric Strategy Forum for medicinal product development for mature B-cell malignancies in children. Eur J Cancer 2019;110:74-85.

42. Thomas A, Teicher BA, Hassan R. Antibody-drug conjugates for cance therapy. Lancet Oncol 2016;17:e254-262.

43. Shingleton JR, Dave SS. Polatuzumab vedotin: honing in on relapsed or refractory diffuse large B-cell Lymphoma. J Clin Oncol 2020;38:166-168.

44. Salas MQ, Climent F, Tapia G, et al. Clinicopathologic features and prognostic significance of CD30 expression in de novo diffuse large B-cell lymphoma (DLBCL): results in a homogeneous series from a single institution. Biomarkers 2020;25:69-75.

45. Broccoli A, Zinzani PL. The unique biology and treatment of primary mediastinal B-cell lymphoma. Best Pract Res Clin Haematol 2018;31: 241-250.

46. Harker-Murray $P$, Leblanc $T$, Mascarin $M$, et al. Response-adapted therapy with nivolumab and brentuximab vedotin (BV), followed by BV and bendamustine for suboptimal response, in children, adolescents, and young adults with standard-risk relapsed/refractory classical Hodgkin lymphoma [abstract]. Blood 2018;132(Suppl):Abstract 927.

47. Cole PD, McCarten KM, Pei Q, et al. Brentuximab vedotin with gemcitabine for paediatric and young adult patients with relapsed or refractory Hodgkin's lymphoma (AHOD1221): a Children's Oncology Group, multicentre single-arm, phase 1-2 trial. Lancet Oncol 2018;19: 1229-1238.

48. Locatelli F, Mauz-Koerholz C, Neville K, et al. Brentuximab vedotin for paediatric relapsed or refractory Hodgkin's lymphoma and anaplastic large-cell lymphoma: a multicentre, open-label, phase 1/2 study. Lancet Haematol 2018;5:e450-e461.

49. Jacobsen ED, Sharman JP, Oki Y, et al. Brentuximab vedotin demonstrates objective responses in a phase 2 study of relapsed/refractory DLBCL with variable CD30 expression. Blood 2015;125:1394-1402.

50. Bartlett NL, Smith MR, Siddiqi T, et al. Brentuximab vedotin activity in diffuse large B-cell lymphoma with CD30 undetectable by visual assessment of conventional immunohistochemistry. Leuk Lymphoma 2017 58:1607-1616.

51. Zinzani PL, Pellegrini C, Chiappella A, et al. Brentuximab vedotin in relapsed primary mediastinal large B-cell lymphoma: results from a phase 2 clinical trial. Blood 2017;129:2328-2330. 
52. Advani A, Coiffier B, Czuczman MS, et al. Safety, pharmacokinetics, and preliminary clinical activity of inotuzumab ozogamicin, a novel immunoconjugate for the treatment of B-cell non-Hodgkin's lymphoma: results of a phase I study. J Clin Oncol 2010;28:2085-2093.

53. Fayad L, Offner F, Smith MR, et al. Safety and clinical activity of a combination therapy comprising two antibody-based targeting agents for the treatment of non-Hodgkin lymphoma: results of a phase I/II study evaluating the immunoconjugate inotuzumab ozogamicin with rituximab. J Clin Oncol 2013;31:573-583.

54. Wagner-Johnston ND, Goy A, Rodriguez MA, et al. A phase 2 study of inotuzumab ozogamicin and rituximab, followed by autologous stem cell transplant in patients with relapsed/refractory diffuse large B-cell lymphoma. Leuk Lymphoma 2015;56:2863-2869.

55. Bhojwani D, Sposto R, Shah NN, et al. Inotuzumab ozogamicin in pediatric patients with relapsed/refractory acute lymphoblastic leukemia. Leukemia 2019;33:884-892.

56. Palanca-Wessels MCA, Czuczman M, Salles G, et al. Safety and activity of the anti-CD79B antibody-drug conjugate polatuzumab vedotin in relapsed or refractory B-cell non-Hodgkin lymphoma and chronic lymphocytic leukaemia: a phase 1 study. Lancet Oncol 2015;16:704-715.

57. Phillips $T$, Brunvand $M$, Chen A, et al. Polatuzumab vedotin combined with obinutuzumab for patients with relapsed or refractory non-Hodgkin lymphoma: preliminary safety and clinical activity of a phase $\mathrm{lb} / \mathrm{II}$ study. Blood 2016;128:622-622.

58. Sehn LH, Herrera AF, Flowers $C R$, et al. Polatuzumab vedotin in relapsed or refractory diffuse large B-cell lymphoma. J Clin Oncol 2020;38: 155-165.

59. Tilly $\mathrm{H}$, Morschhauser $\mathrm{F}$, Bartlett NL, et al. Polatuzumab vedotin in combination with immunochemotherapy in patients with previously untreated diffuse large B-cell lymphoma: an open-label, nonrandomised, phase 1b-2 study. Lancet Oncol 2019;20:998-1010.

60. Morschhauser F, Flinn IW, Advani R, et al. Polatuzumab vedotin or pinatuzumab vedotin plus rituximab in patients with relapsed or refractory non-Hodgkin lymphoma: final results from a phase 2 randomised study (ROMULUS). Lancet Haematol 2019;6:e254-265.

61. Rader C. Bispecific antibodies in cancer immunotherapy. Curr Opin Biotechnol 2019;65:9-16.

62. Topp MS, Gökbuget N, Stein AS, et al. Safety and activity of blinatumomab for adult patients with relapsed or refractory B-precursor acute lymphoblastic leukaemia: a multicentre, single-arm, phase 2 study. Lancet Oncol 2015;16:57-66.

63. Gore L, Locatelli F, Zugmaier G, et al. Survival after blinatumomab treatment in pediatric patients with relapsed/refractory B-cell precursor acute lymphoblastic leukemia. Blood Cancer J 2018;8:80.

64. von Stackelberg A, Locatelli F, Zugmaier G, et al. Phase I/phase II study of blinatumomab in pediatric patients with relapsed/refractory acute lymphoblastic leukemia. J Clin Oncol 2016;34:4381-4389.

65. Kantarjian H, Stein A, Gökbuget N, et al. Blinatumomab versus chemotherapy for advanced acute lymphoblastic leukemia. N Engl J Med 2017;376:836-847.

66. Viardot A, Goebeler ME, Hess G, et al. Phase 2 study of the bispecific T-cell engager (BiTE) antibody blinatumomab in relapsed/refractory diffuse large B-cell lymphoma. Blood 2016;127:1410-1416.

67. Bartlett NL, Assouline SE, Sehn LN, et al. Managing cytokine release syndrome (CRS) and neurotoxicity with step-fractionated dosing of mosunetuzumab in relapsed/refractory (R/R) B-cell non-Hodgkin lymphoma (NHL) [abstract]. J Clin Oncol 2019;37(Suppl):Abstract 7518.

68. Schuster SJ, Bartlett NL, Assouline S, et al. Mosunetuzumab induces complete remissions in poor prognosis non-Hodgkin lymphoma patients, including those who are resistant to or relapsing after chimeric antigen receptor T-cell (CAR-T) therapies, and is active in treatment through multiple lines [abstract]. Blood 2019;134(Suppl):Abstract 6.

69. Bannerji R, Allan JN, Arnason JE, et al. Clinical activity of REGN1979, a bispecific human, anti-CD20 $x$ anti-CD3 antibody, in patients with relapsed/refractory (R/R) B-cell non-Hodgkin lymphoma (B-NHL) [abstract]. Blood 2019;134(Suppl):Abstract 762.

70. Barsan $\mathrm{V}$, Ramakrishna S, Davis KL. Immunotherapy for the treatment of acute lymphoblastic leukemia. Curr Oncol Rep 2020;22:11.

71. Vairy S, Garcia JL, Teira P, et al. CTL019 (tisagenlecleucel): CAR-T therapy for relapsed and refractory B-cell acute lymphoblastic leukemia. Drug Des Devel Ther 2018;12:3885-3898.

72. Schuster SJ, Bishop MR, Tam CS, et al. Tisagenlecleucel in adult relapsed or refractory diffuse large B-cell lymphoma. N Engl J Med 2019; $380: 45-56$
73. Neelapu SS, Locke FL, Bartlett NL, et al. Axicabtagene ciloleucel CAR T-cell therapy in refractory large B-cell lymphoma. N Engl J Med 2017; 377:2531-2544.

74. Bachier CR, Palomba ML, Abramson JS, et al. Outpatient treatment with lisocabtagene maraleucel (liso-cel) in three ongoing clinical studies in relapsed/refractory (R/R) B cell non-Hodgkin lymphoma (NHL), including second-line transplant ineligible patients: Transcend NHL 001, Outreach, and PILOT [abstract]. Blood 2019;134(Suppl):Abstract 2868.

75. Shadman M, Gopal AK, Smith SD, et al. CD20 targeted CAR-T for highrisk B-cell non-Hodgkin lymphomas [abstract]. Blood 2019;134(Suppl): Abstract 3235

76. Jacobson CA, Herrera AF, Budde LE, et al. Initial findings of the phase 1 trial of PBCAR0191, a CD19 targeted allogeneic CAR-T cell therapy [abstract]. Blood 2019;134(Suppl):Abstract 4107

77. Ying $Z$, Huang $X F$, Xiang $X$, et al. A safe and potent anti-CD19 CAR T cell therapy. Nat Med 2019;25:947-953.

78. Zhang W, Hu B, Jing L, et al. Early response observed in pediatric patients with refractory/relapsed B-cell non-Hodgkin lymphoma treated with sequential chimeric antigen receptor T cells [abstract]. Blood 2019 134(Suppl):Abstract 1945.

79. Rivers J, Annesley C, Summers C, et al. Early response data for pediatric patients with non-Hodgkin lymphoma treated with CD19 chimeric antigen receptor (CAR) T-cells [abstract]. Blood 2018;132(Suppl):Abstract 2957

80. Wilson $\mathrm{WH}$, Young RM, Schmitz R, et al. Targeting B cell receptor signaling with ibrutinib in diffuse large B cell lymphoma. Nat Med 2015; 21:922-926.

81. Younes A, Sehn LH, Johnson $P$, et al. Randomized phase III trial of ibrutinib and rituximab plus cyclophosphamide, doxorubicin, vincristine and prednisone in non-germinal center B-cell diffuse large B-cell lymphoma. J Clin Oncol 2019:37:1285-1295.

82. Lunning M, Vose J, Nastoupil L, et al. Ublituximab and umbralisib in relapsed/refractory B-cell non-Hodgkin lymphoma and chronic lymphocytic leukemia. Blood 2019;134:1811-1820.

83. Bhatti $M$, Ippolito $T$, Mavis $C$, et al. Pre-clinical activity of targeting the PI3K/Akt/mTOR pathway in Burkitt lymphoma. Oncotarget 2018;9: 21820-21830.

84. Kahl BS, Byrd J, Flinn IW. Clinical safety and activity in a phase 1 study of CAL-101, an isoform-selective inhibitor of phosphatidylinositol 3-kinase $\mathrm{P} 110 \delta$, in patients with relapsed or refractory non-Hodgkin lymphoma [abstract]. Blood 2010;116(Suppl):Abstract 1777

85. Davids MS, Roberts AW, Seymour JF, et al. Phase I first-in-human study of venetoclax in patients with relapsed or refractory non-Hodgkin lymphoma. J Clin Oncol 2017;35:826-833.

86. Zelenetz AD, Salles G, Mason KD, et al. Venetoclax plus R- or G-CHOP in non-Hodgkin lymphoma: results from the CAVALLI phase $1 \mathrm{~b}$ trial. Blood 2019;133:1964-1976.

87. de Vos S, Swinnen LJ, Wang D, et al. Venetoclax, bendamustine, and rituximab in patients with relapsed or refractory $\mathrm{NHL}$ : a phase lb dosefinding study. Ann Oncol 2018;29:1932-1938.

88. Nowakowski GS, Chiappella A, Witzig TE, et al. ROBUST: lenalidomide$\mathrm{R}-\mathrm{CHOP}$ versus placebo-R-CHOP in previously untreated $\mathrm{ABC}$-type diffuse large B-cell lymphoma. Future Oncol 2016;12:1553-1563.

89. Leonard JP, Trneny M, Izutsu K, et al. AUGMENT: a phase III study of lenalidomide plus rituximab versus placebo plus rituximab in relapsed or refractory indolent lymphoma. J Clin Oncol 2019;37:1188-1199.

90. Nowakowski GS, Chiappella A, Witzig TE, et al. ROBUST: lenalidomide$\mathrm{R}-\mathrm{CHOP}$ versus placebo-R-CHOP in previously untreated $\mathrm{ABC}$-type diffuse large B-cell lymphoma. Future Oncol 2016;12:1553-1563.

91. Nowakowski GS, LaPlant B, Macon WR et al. Lenalidomide combined with R-CHOP overcomes negative prognostic impact of non-germinal center B-cell phenotype in newly diagnosed diffuse large B-cell lymphoma: a phase II study. J Clin Oncol 2015;33:251-257.

92. Lin Z, Chen X, Li Z, et al. The role of bortezomib in newly diagnosed diffuse large B cell lymphoma: a meta-analysis. Ann Hematol 2018;97: 2137-2144.

93. Hu B, Jacobs R, Ghosh N. Checkpoint inhibitors Hodgkin lymphoma and non-Hodgkin lymphoma. Curr Hematol Malig Rep 2018;13:543-554.

94. Zinzani PL, Santoro A, Gritti G, et al. Nivolumab combined with brentuximab vedotin for relapsed/refractory primary mediastinal large B-cel lymphoma: efficacy and safety from the phase II CheckMate 436 study. J Clin Oncol 2019;37:3081-3089.

95. Armand P, Rodig S, Melnichenko V, et al. Pembrolizumab in relapsed or refractory primary mediastinal large B-cell lymphoma. J Clin Oncol 2019; 37:3291-3299. 
96. Kiyasu J, Miyoshi $\mathrm{H}$, Hirata $\mathrm{A}$, et al. Expression of programmed cell death ligand 1 is associated with poor overall survival in patients with diffuse large B-cell lymphoma. Blood 2015;126:2193-2201.

97. Ansell SM, Minnema MC, Johnson P, et al. Nivolumab for relapsed/ refractory diffuse large B-cell lymphoma in patients ineligible for or having failed autologous transplantation: a single-arm, phase II study. J Clin Oncol 2019;37:481-489.

98. Smith SD, Till BG, Shadman MS, et al. Pembrolizumab with R-CHOP in previously untreated diffuse large B-cell lymphoma: potential for biomarker driven therapy. Br J Haematol 2020;189:1119-1126.

99. Smith MA, Altekruse SF, Adamson PC, et al. Declining childhood and adolescent cancer mortality. Cancer 2014;120:2497-2506.
100. Gerrard M, Cairo MS, Weston C, et al. Excellent survival following two courses of COPAD chemotherapy in children and adolescents with resected localized B-cell non-Hodgkin's lymphoma: results of the FAB/LMB 96 international study. Br J Haematol 2008;141: 840-847.

101. Cairo MS, Gerrard M, Sposto R, et al. Results of a randomized international study of high-risk central nervous system B non-Hodgkin lymphoma and B acute lymphoblastic leukemia in children and adolescents. Blood 2007;109:2736-2743.

102. Dang NH, Ogura M, Castaigne $\mathrm{S}$, et al. Randomized, phase 3 trial of inotuzumab ozogamicin plus rituximab versus chemotherapy plus rituximab for relapsed/refractory aggressive B-cell non-Hodgkin lymphoma. Br J Haematol 2018;182:583-586.

\title{
ENROLL NOW
}

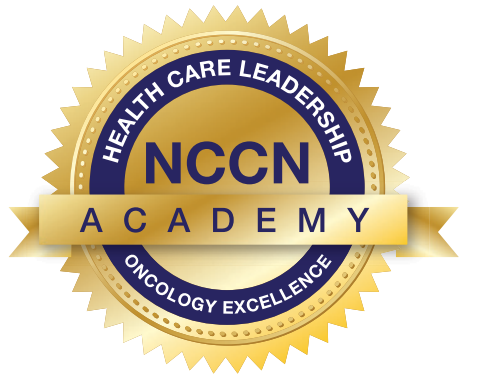

\section{NCCN Academy for Excellence \& Leadership in Oncology ${ }^{\mathrm{Tm}}$}

\section{School of Pharmaceutical \& Biotech Business}

\author{
VIRTUAL PROGRAM \\ Tuesday, October 6 | 12:30 - 3:30 PM ET \\ Module I: Oncology Medical Home: Perspectives on \\ Evolution, Impact \& Future State
}

\section{Module II: Pathways: A View Across Developers and Stakeholders}

This program provides pharmaceutical and biotechnology professionals with the significant opportunity to view the oncology space and its current and future operational issues from providers, payers, patient advocates, and other stakeholders.

\section{Enroll Today: NCCN.org/academy/virtual-october}

